\title{
BI DIRECTIONAL SPEED CONTROL OF DC MOTOR AND STEPPER MOTOR THROUGH MAT LAB USING PIC MICRO CONTROLLER
}

\author{
Ozwin Dominic Dsouza ${ }^{1}$, Manjunathababu. $\mathbf{P}^{2}$, Babu Naik ${ }^{3}$ \\ ${ }^{1}$ BMS Institute of Technology, Bangalore, India \\ ozwindsouza@gmail.com,manjubabup@gmail.com
}

\begin{abstract}
In any industry speed control of an electric drive system is very critical and crucial. Every designer aims at achieving a control methodology having high degree of precision. But industry needs are ever evolving in nature. Hence it is very much essential that along with conventional speed control mechanisms we must also have simple interactive graphical based control strategies. Several algorithms/methodologies have been developed over the years to achieve speed control of motors. In this context by encompassing the usability of Mat Lab, work has been done to control the speed of stepper motor and DC motor using microcontroller. Microcontroller is programmed to achieve bi directional speed control. The main objective of this work is to develop the graphical user interface of motor control through mat Lab guide and the interface of the same with hardware via serial communication. PIC is used as the controller.
\end{abstract}

Keywords-DC, PIC, $\mu C, A C, G U I, I C$ $* * *$

\section{INTRODUCTION}

DC motors are widely used in adjustable speed drives and position control applications. In DC motor speeds below its base speed is controlled by armature voltage control method [1] [2]. Speeds above base speed are achieved by encompassing field flux control method. Speed controls of DC motors are much cheaper and simpler as compared to AC motors. This is mainly because in DC motor speed control only two control parameters are sufficient (voltage or flux). On the contrary an AC motor speed control requires three control parameters.

A stepper motor is a marvel in simplicity [1]. It is basically an electromechanical device which converts electrical pulses into discrete mechanical movements. Unlike DC motors it has no brushes and contacts and is a synchronous motor with the magnetic field electronically switched to rotate the armature magnet. The essential function of a stepper motor is to translate switching excitation changes into precisely defined increments of rotor position. A stepper motor can divide a full rotation into a large number of steps, for example 200 steps. The motor thus can be made to rotate through a precise angle. The sequence of applied pulses is directly related to the direction of motor rotation. There are different types of stepper motors; namely (i) permanent magnet (ii) variable reluctance (iii) Hybrid motors. The basic block diagram of stepper motor is shown in figure 1 below.

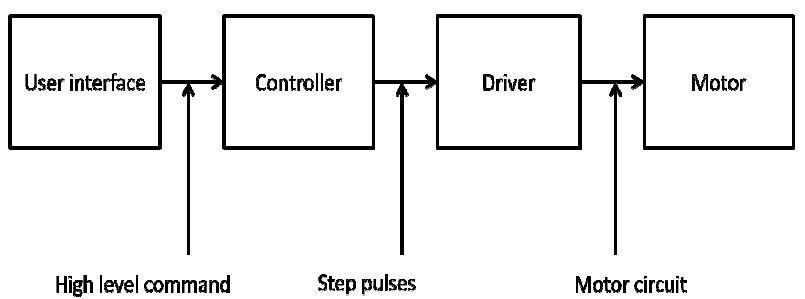

Fig 1 Block diagram of stepper motor

Speed control of DC motor and stepper motor through Mat Lab using PIC microcontroller $(\mu \mathrm{C})$ [3] is an initiative taken to emphasize the use of two types of motors( stepper and DC motor) together. PIC $\mu \mathrm{C}$ used here enables the user to code in such a way that the DC and stepper motors rotate in forward and reverse direction at any given point of time as and when specified by the user through keys. At the same time user has an option of manual intervention in direction of rotation, as PIC $\mu \mathrm{C}$ is designed to operate in both auto and manual mode.

It is a proven fact that proven over the years that MAT Lab is a powerful design/simulation tool. It gives greater flexibility to a designer to analyze the circuit/product before the actual implementation. Signals required to run in auto mode are generated through Mat Lab using Graphical user interface (GUI) [4]. GUI facilitates the simple coding technique through which signals could be sent. This work describes the GUI codes for sending signals to hardware through parallel port under the supervision of $\mu \mathrm{C}$. This type of speed control approach is highly useful in applications where precise speed control is required for example in computer numeral control (CNC). 


\section{HARDWARE DESCRIPTION}

Detailed block diagram of the project is as shown in figure 2 below. It mainly consists of the following parts.

\section{A. DC Motor}

A dc motor works based on the principle of electromagnetic induction Here a DC motor having 9-12V, $2000 \mathrm{RPM}$ rating is used. DC motor is connected to PIC $\mu \mathrm{C}$ via a dual $\mathrm{H}$-Bridge motor driver integrated circuit. Motor drivers acts as current amplifiers. They take low-current control signal and provide high current signals. The higher current signals eventually drive the motor in the designed program sequence.

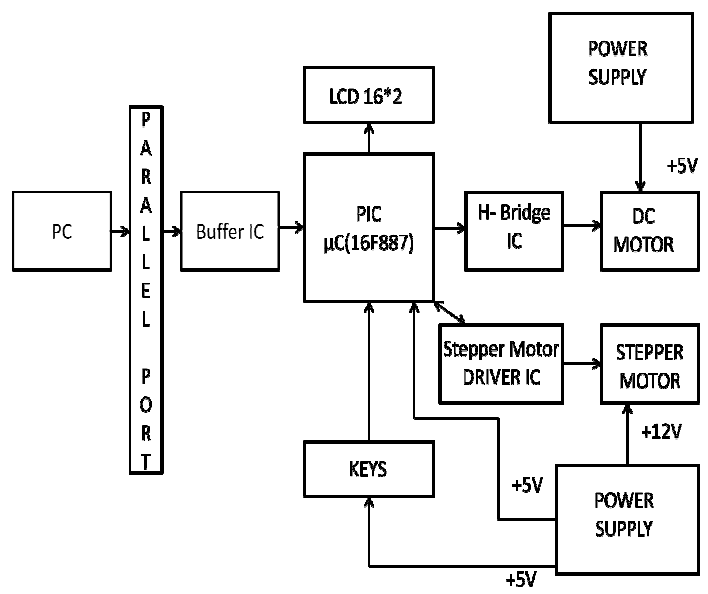

Fig 2 Block diagram of the project

\section{B. Stepper Motor:}

A stepper motor having specification $12 \mathrm{~V}, 1.8$ Degrees is used here. It is connected to PIC $\mu \mathrm{C}$ via stepper motor driver IC. This IC basically takes the control signals from $\mu \mathrm{C}$ and in turn takes controlling actions on stepper motor.

\section{Buffer IC:}

Buffer IC is also called as line driver IC. Buffer IC passes the signal from one circuit to the other without any changes in signals. The operating voltage rages from $2 \mathrm{~V}$ to $6 \mathrm{~V}$.

\section{H-Bridge Motor Control Circuit:}

A very popular/simpler way of achieving speed control of a DC motor is by using a single transistor based control. But the main disadvantage of this control strategy is that, only uni-directional speed control is possible. But in many applications it is necessary to operate the motor in both directions; clockwise or anticlockwise. A very good way of achieving this is to connect the motor to a Transistor H-Bridge circuit. With this arrangement Bi-directional DC motor operation becomes possible.

\subsection{Basic Bi-directional H-bridge Circuit:}

Figure 3 shows the basic H-bridge circuit. It is named so because the basic configuration of the four switches, transistors resembles the letter $\mathrm{H}$ with the motor positioned in the center. The transistor or MOSFET H-bridge is probably the most commonly used Bi-directional DC motor control circuit. Which uses complementary transistor pairs, both NPN and PNP in each branch along with motor. The transistor operates in pairs to control the motor.

From figure 3, control input $\mathrm{A}$, operates the motor in one direction; Forward rotation and input B operates the motor in Reverse direction; Reverse rotation. Switching the transistors ON or OFF in their diagonal pairs results in bi-directional control of the motor. Then transistor TR1 is ON and transistor TR2 is OFF, point A is connected to the supply voltage (+Vcc) and if transistor TR3 is OFF

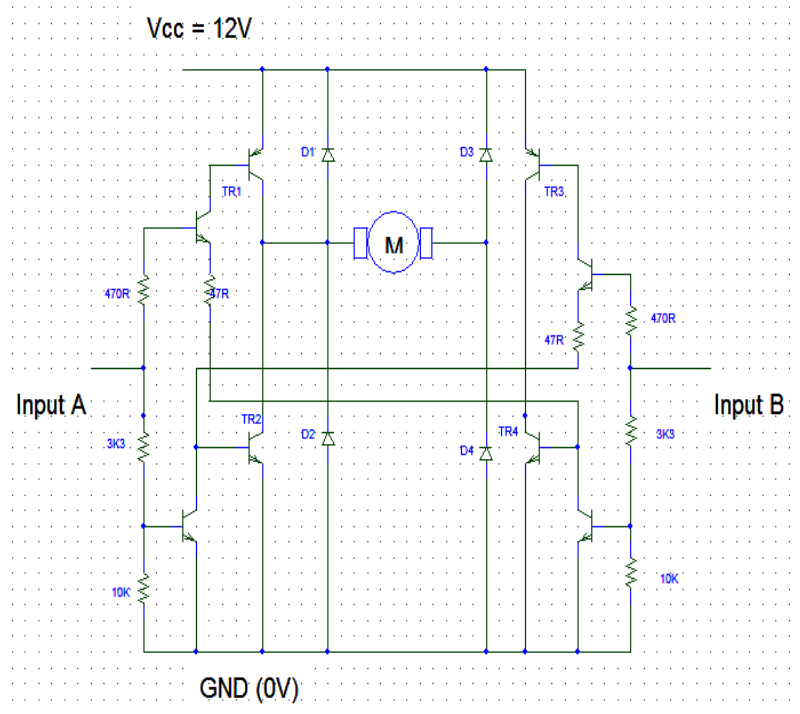

Fig 3 H-Bridge circuit

H-Bridge circuit and transistor TR2 is OFF, point $\mathrm{A}$ is connected to the supply voltage $(+\mathrm{Vcc})$ and if transistor TR3 is $\mathrm{OFF}$ and transistor TR4 is ON, point B is connected to ground (GND) terminal. Then the motor will rotate in one direction corresponding to motor terminal A being positive and terminal $\mathrm{B}$ being negative. If the switching states are reversed, then the motor will operate in the reverse direction. The H-bridge truth table for the motor operation is shown in Table 1.

It is important to note that Input A and Input B must not be kept at High Logic. If not then the power supply terminals gets short circuited and the fuse blows. IC L293D is used in this work as motor driver IC. This IC is a dual $\mathrm{H}$-bridge motor driver, so with one IC, user can interface two DC motors which can be controlled both in forward and reverse direction. For protecting 
the circuit from back emf output diodes are included within the IC. This has made it a best choice for DC motor driver. Pin configuration is shown in figure 4 .

Table 1 H-Bridge truth table

\begin{tabular}{|l|l|l|}
\hline $\begin{array}{l}\text { Input A (TR1 } \\
\text { and TR4) }\end{array}$ & $\begin{array}{l}\text { Input B (TR2 } \\
\text { and TR3) }\end{array}$ & Motor Function \\
\hline 0 & 0 & $\begin{array}{l}\text { Motor } \\
\text { Stops(OFF) }\end{array}$ \\
\hline 1 & 0 & $\begin{array}{l}\text { Motor rotes } \\
\text { forward }\end{array}$ \\
\hline 0 & 1 & $\begin{array}{l}\text { Motor rotates } \\
\text { reverse }\end{array}$ \\
\hline 1 & 1 & Not Allowed \\
\hline
\end{tabular}

\subsection{Stepper Motor Driver IC:}

This IC is used to drive the current from the $\mu \mathrm{C}$ to the stepper motor. Driver IC used here is ULN2803. It consists of eight Darlington transistors with common emitters and integral suppression diodes for inductive loads. Input to this circuit is given from the PORT of the

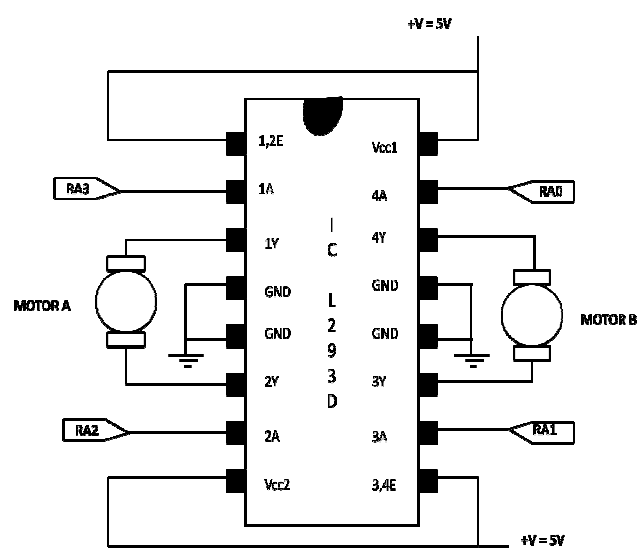

Fig 4 Pin configuration

$\mu \mathrm{C}$. Output of this IC is given to stator of stepper motor.

\subsection{PIC microcontroller $(\mu \mathrm{C})$ :}

PIC16F887 is used in this work. This $\mu \mathrm{C}$ encompasses RISC architecture. Operating frequency is $0-20 \mathrm{MHz}$. PIC $\mu \mathrm{C}$ performs the job of a controller. This $\mu \mathrm{C}$ totally controls the entire process. It is coded in such a fashion that both manual and automatic speed control is possible.

\section{SOFTWARE DESCRIPTION}

Software part of this project consists of two parts (1) Coding of $\mu \mathrm{C}$ (2) Creating GUI for speed control. Unique approach of this project is that the GUI creation and the controlling through it.
Mat Lab supports wide range of applications, including signal and image processing, communications, control design, test and measurement etc.

GUI is a pictorial interface to a program. A good GUI can make programs easier to use by providing them with a descriptive appearance and with insightful controls like pushbuttons, list boxes, sliders, menus so on and so forth. The GUI should behave in an understandable and knowable manner, so that a user knows what to anticipate when performs an action example, like mouse click occurs on a pushbutton. The GUI should initiate the action described on the label of the button. Figure 5 shows the developed GUI for motor control.

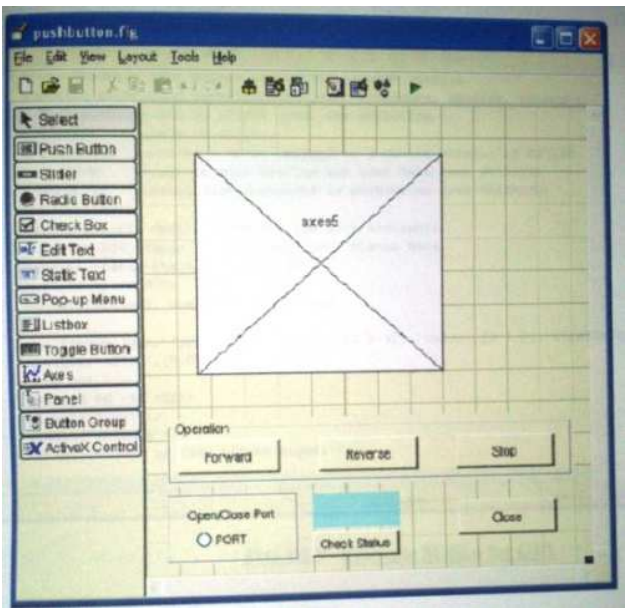

Fig 5 GUI of Motor Control

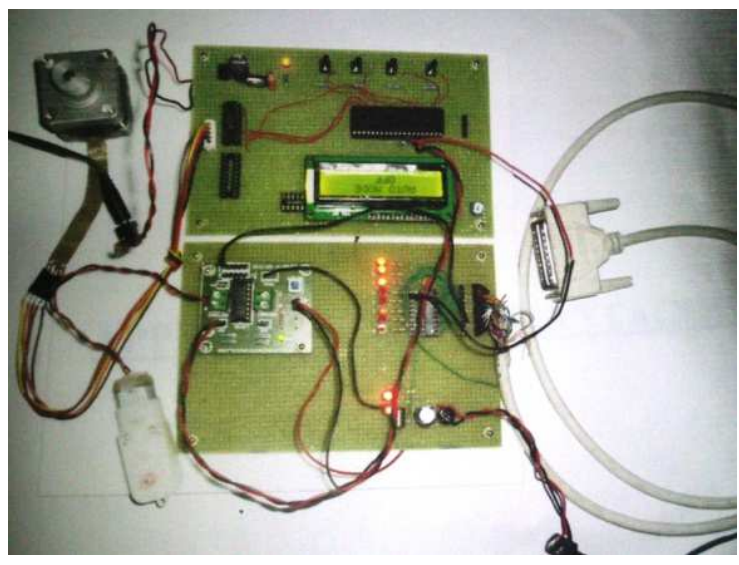

Fig 6 Actual implementation of Motor Control

After laying out the GUI component and setting the property, the GUI will look like as shown in figure 5. Finally ode is written to implement the behavior associated with each call beck function. This kind of programming is often called eventdriven programming. Actual view of the project is given in figure 6 . 


\section{CONCLUSIONS}

In this work a computer program has been developed for programming and driving stepper and DC motors via the parallel port of a PC; a GUI performing all defined motions individually are also improved. In addition, the GUI allows programmers to define the user defined functions. So, the programmers can make stepper motors and DC motors to rotate in all manners. The developed programs are tested with a stepper motor of 1.80 step angle in forward and reverse with speed variation modes. Also the developed program is tested with a DC motor for forward and reverse motion control. Very satisfactory results are obtained from experimental setup. The program can be used in applications containing steeper and DC motors performing complex motions. From the obtained results it is concluded that speed control of DC motor and Stepper motor can be used in robotic systems, DC drive systems, paper printing mills, CNC machines.

\section{FUTURE WORK}

As a future expansion, work is under progress, so as to control the motors using sensor less technique using faster $\mu \mathrm{C}$ or DSP. Work is also under progress in the control software loop. Design of Fuzzy control, neural network control, Adaptive control algorithms are currently under progress.

\section{ACKNOWLEDGMENT}

The authors would like to thank Dr. S.C. Sharma, Chief Mentor BMSIT and BMSSA for providing constant thrust/ inspiration to achieve more. The authors also thank Prof. S. Venkateswaran Principal BMSIT for his constant support. The efforts of final year Electrical students while realizing this work is also acknowledged.

\section{REFERENCES}

[1] A. E. Fitzgerald, Charles Kingsley Jr, Stephen Umans "Electric Machinery" McGraw-Hill Higher Education, Sixth Edition

[2] Bimal K Bose, "Power Electronics And Motor Drives: Advances and Trends". Academic Press, 2006 edition

[3] J. Petrovciv, S. Strmcnik,"A microcomputer based speed controller for lift drives” IEEE Trans. Ind.Appl.,24(3), vol.34, pp.487-498,1998.

[4] Mat Lab, Creating Graphical User Interfaces, Version 7, The Mathworks Inc. Naticks/Ma, 2006.

\section{BIOGRAPHIES}

Ozwin Dominic Dsouza has received his masters from National Institute of Engineering, Mysore, India. Currently working as Assistant professor in the department of Electrical and Electronics Engg at BMSIT Bangalore. His research interests are in the field of electric drives and systems and control systems, converter topologies and electrical machines.
Manjunatha Babu $\mathbf{P}$ obtained his B.E and M.E from Visvesvaraya Technological University, Bangalore University. Presently working as Assistant Professor in the Dept of Electrical and Electronics Engineering at BMS Institute of Technology, Bangalore, India. His interests are in the area of power and Energy systems. Power System Operation and Control, Power Transmission and Distributions System Studies, FACTS Controllers.

Babu Naik Gugulothu, has received his masters from the Indian Institute of Science (IISc) Bangalore, India. Currently working as Assistant professor in the department of Electrical and Electronics Engg at BMSIT Bangalore. His research interests are in the field of Industrial drives and applications, power systems, SMPS circuits, converter topologies and electrical machines. 